\title{
Deferoxamine retinopathy: spectral domain-optical coherence tomography findings
}

\author{
Cheng-Hsiu Wu', Chao-Ping Yang ${ }^{2,3}$, Chi-Chun Lai ${ }^{1,3}$, Wei-Chi Wu ${ }^{1,3}$ and Yi-Hsing Chen ${ }^{1,3^{*}}$
}

\begin{abstract}
Background: To describe the spectral domain optical coherence tomography (SD-OCT) findings of a patient who developed pigmentary retinopathy following high-dose deferoxamine administration.

Case presentation: A 34-year-old man with thalassemia major complained of nyctalopia and decreased vision following high-dose intravenous deferoxamine to treat systemic iron overload. Fundus examination revealed multiple discrete hypo-pigmented lesions at the posterior pole and mid-peripheral retina. Recovery was partial following cessation of desferrioxamine six weeks later. A follow-up SD-OCT showed multiple accumulated hyper-reflective deposits primarily in the choroid, retina pigment epithelium (RPE), and inner segment and outer segment (IS/OS) junction.

Conclusion: Deferoxamine retinopathy primarily targets the RPE-Bruch membrane-photoreceptor complex, extending from the peri-fovea to the peripheral retina with foveola sparing. An SD-OCT examination can serve as a simple, noninvasive tool for early detection and long-term follow-up.
\end{abstract}

Keywords: Spectral domain optical coherence tomography, Deferoxamine, Retinopathy

\section{Background}

Deferoxamine is an iron-chelating agent used to treat chronic iron overload in patients with thalassemia major and other hematologic conditions requiring routine blood transfusion [1,2]. The incidence of deferoxamine-related ocular toxicity is approximately $1.2 \%$ based on a prior study [3]. The clinical presentations may include night blindness, centrocaecal scotoma, constricted peripheral visual field, pigmentary retinopathy, and optic neuropathy [3]. Retinal pigmentary change was most frequently reported [4]. This case report pathologically characterizes the spectral domain optical coherence tomography (SD-OCT, SPECTRALIS SD-OCT, Heidelberg, Germany) and nearinfrared reflectance (NIR) findings in a patient with deferoxamine retinopathy.

\section{Case presentation}

A 34-year-old Taiwanese man with beta-thalassemia major had been administered routine blood transfusion and

\footnotetext{
* Correspondence: yihsing@gmail.com

'Department of Ophthalmology, Chang Gung Memorial Hospital, No. 5, Fu-Hsing Street, Kweishan, Taoyuan 333, Taiwan

${ }^{3}$ College of Medicine, Chang Gung University, Taoyuan, Taiwan

Full list of author information is available at the end of the article
}

subcutaneous deferoxamine at $30 \mathrm{mg} / \mathrm{kg} /$ day for 20 years since youth. He was hospitalized for a compression fracture and myelopathy of the thoracic spine. He presented with acute onset of decreased vision, impaired color vision, and night blindness following continuous intravenous deferoxamine $(98 \mathrm{mg} / \mathrm{kg})$ for 42 days for the treatment of elevated serum ferritin level. On ophthalmic examinations, the bestcorrected vision was 20/200 in the right eye and 20/40 in the left eye. The intraocular pressure measurement and anterior segment examination yielded normal results for both eyes. The fundus examination revealed multiple discrete hypo-pigmented circular lesions over the posterior pole and mid-peripheral retina in both eyes.

Deferoxamine retinopathy was suspected, and the patient was switched to oral deferasirox/deferiprone. Six weeks later, there was an improvement in the best-corrected vision (20/60 in the right eye and 20/25 in the left eye) and color vision. Retinal pigmentary changes became confluent (Figure 1). NIR showed hyper-reflective deposits particularly in the parafoveal and perifoveal areas (Figure 2). SDOCT showed multiple confluent hyper-reflective deposits in the choroid, retinal pigment epithelium (RPE) and IS/OS junction. Thickened RPE, Bruch's membrane, and choroid space were also discovered. The IS/OS junction was most 


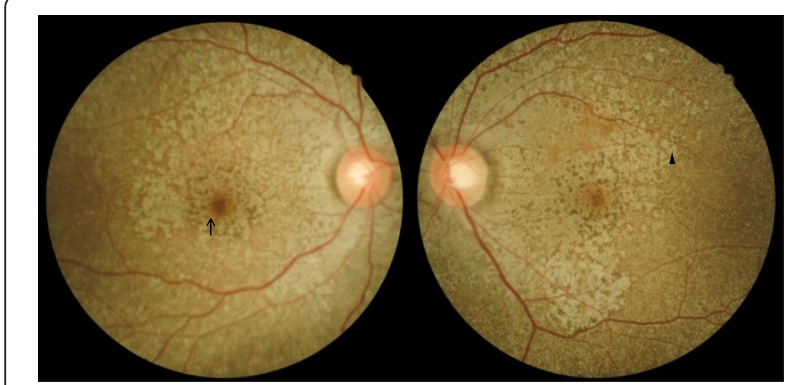

Figure 1 Dilated fundus examination at the 6-week follow-up visit. A dilated fundus examination revealed diffuse and confluent hypo-pigmented pinpoint lesions extending from the posterior pole (arrow) to the peripheral retina (arrowhead).

severely disrupted at the perifoveal and parafoveal areas than at the foveola area (Figure 3).

\section{Conclusion}

Deferoxamine is a widely used chelating agent in treating transfusional hemochromatosis $[1,5]$. Visual symptoms included decreased visual acuity, night blindness, and colour vision abnormalities [2-6]. These ophthalmic examination findings have been reported extensively. Sumu et al. observed speckled hyper-fluorescence with well-demarcated areas of blocked fluorescence on fluorescein angiography [6]. Markedly reduced photopic, scotopic, and $30-\mathrm{Hz}$ flicker response amplitudes were reported on electroretinograms. Electro-oculogram typically showed reduced light-peak to dark-trough ratios $[4,6]$. Viola et al. reported abnormal fundus autofluorescence in 9\% of 197 patients, but only 5 patients reported visual symptoms [7]. Viola et al. further described the pattern dystrophy-like or minimal changes of macular lesions in patients with deferoxamine retinopathy by using NIR and SD-OCT which pointed out the disease itself affects the RPE-Bruch membrane-photoreceptor complex [8].

The pathophysiology of deferoxamine-related retinopathy has been investigated for several years. Rahi et al. first

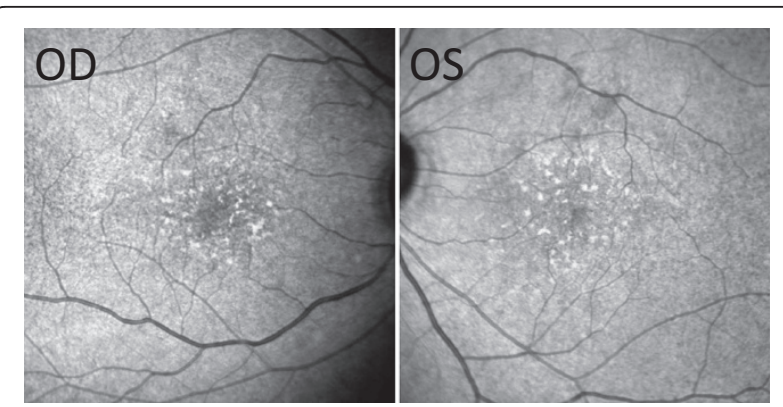

Figure 2 Near-infrared reflectance (NIR) at the 6-week re-examination. NIR showed hyper-reflective deposits particularly in the parafoveal and perifoveal areas.

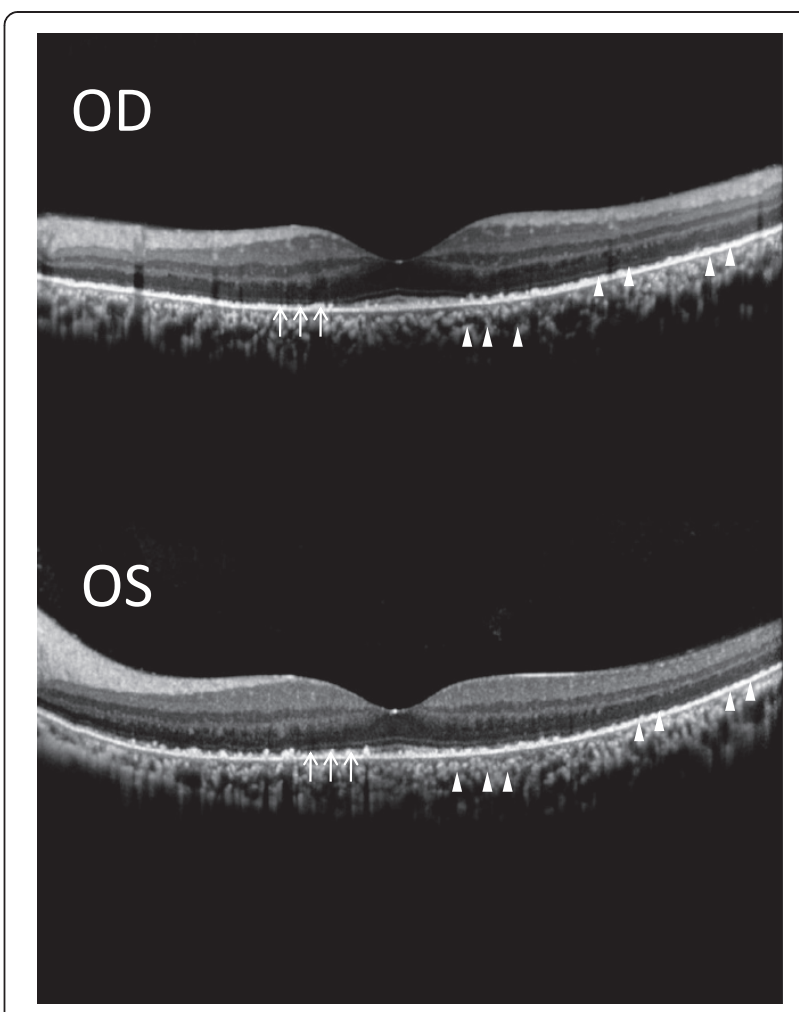

Figure 3 SD-OCT at the 6-week re-examination. SD-OCT revealed a disrupted IS/OS junction (arrow) and multiple diffuse and confluent hyper-reflective deposits in the retinal pigment epithelium (RPE), IS/OS junction, and choroid (arrowhead). Thickened RPE, Bruch's membrane, and choroidal space were also observed.

reported electron microscopic findings of deferoxamine retinopathy, including patchy RPE depigmentation, abnormally thickened Bruch's membrane, and normal photoreceptors [9]. Previous studies also discovered that iron overload and iron-chelating agents both may be mutually confounding factors in the causation of ocular changes of thalassemia such as RPE mottling [5,10-13]. The SD-OCT findings in our case revealed multiple confluent hyperreflective deposits in the RPE, IS/OS junction and choroid (Figure 3). We hypothesized that hyper-reflective deposits detected by means of SD-OCT may represent a primarily involvement of RPE-Bruch membrane-photoreceptor complex in deferoxamine toxicity which correlated with previous histologic findings $[8,9]$.

Ocular deferoxamine toxicity could cause vision impairment; regular ophthalmic assessment is required in these patients. We presented the SD-OCT findings of deferoxamine retinopathy highly correlated with previous histologic descriptions and showed that the toxicity primarily involved the RPE-Bruch membrane-photoreceptor complex. Noninvasive SD-OCT and NIR imaging, both well tolerated by patients, may be helpful in early detection and long-term monitoring. 


\section{Consent}

The patient provided written informed consent for the publication of this case report and any accompanying images. A copy of the written consent is available for editorial review.

\section{Abbreviations}

SD-OCT: Spectral domain optical coherence tomography; IS/OS: Inner segment/outer segment; RPE: Retinal pigment epithelium.

\section{Competing interests}

The authors declare that they have no competing interests.

\section{Authors' contributions}

C-HW conducted the literature search and composed the manuscript. Y-HC conceived the idea for the manuscript, conducted a literature search, and critiqued the manuscript. All authors read and approved the final manuscript.

\section{Acknowledgements}

The authors thank Dr. Chao-Ping Yang from the Department of Pediatrics, Chang Gung Memorial Hospital, in Taoyuan, Taiwan for her expertise in managing this patient.

\section{Author details}

'Department of Ophthalmology, Chang Gung Memorial Hospital, No. 5, Fu-Hsing Street, Kweishan, Taoyuan 333, Taiwan. ${ }^{2}$ Department of Pediatrics, Chang Gung Memorial Hospital, Taoyuan, Taiwan. ${ }^{3}$ College of Medicine, Chang Gung University, Taoyuan, Taiwan.

Received: 21 March 2014 Accepted: 26 June 2014

Published: 2 July 2014

\section{References}

1. Kontoghiorghes GJ, Pattichi K, Hadjigavriel M, Kolnagou A: Transfusional iron overload and chelation therapy with deferoxamine and deferiprone (L1). Transfus Sci 2000, 23(3):211-223.

2. Lakhanpal V, Schocket SS, Jiji R: Deferoxamine (Desferal)-induced toxic retinal pigmentary degeneration and presumed optic neuropathy. Ophthalmol 1984, 91(5):443-451.

3. Baath JS, Lam WC, Kirby M, Chun A: Deferoxamine-related ocular toxicity: incidence and outcome in a pediatric population. Retina 2008, 28(6):894-899.

4. Haimovici R, D'Amico DJ, Gragoudas ES, Sokol S: Deferoxamine Retinopathy Study G: The expanded clinical spectrum of deferoxamine retinopathy. Ophthalmology 2002, 109(1):164-171.

5. Arora A, Wren S, Gregory Evans K: Desferrioxamine related maculopathy: a case report. Am J Hematol 2004, 76(4):386-388.

6. Simon S, Athanasiov PA, Jain R, Raymond G, Gilhotra JS: Desferrioxaminerelated ocular toxicity: a case report. Indian J Ophthalmol 2012, 60(4):315-317.

7. Viola F, Barteselli G, Dell'arti L, Vezzola D, Villani E, Mapelli C, Zanaboni L, Cappellini MD, Ratiglia R: Abnormal fundus autofluorescence results of patients in long-term treatment with deferoxamine. Ophthalmol 2012, 119(8):1693-1700.

8. Viola F, Barteselli G, Dell'arti L, Vezzola D, Mapelli C, Villani E, Ratiglia R: Multimodal Imaging in Deferoxamine Retinopathy. Retina 2013, 0:1-11.

9. Rahi AH, Hungerford $\mathrm{JL}$, Ahmed Al: Ocular toxicity of desferrioxamine: light microscopic histochemical and ultrastructural findings. $\mathrm{Br} J$ Ophthalmol 1986, 70(5):373-381.

10. Taneja R, Malik P, Sharma M, Agarwal MC: Multiple transfused thalassemia major: ocular manifestations in a hospital-based population. Indian J Ophthalmol 2010, 58(2):125-130.

11. Blake DR, Winyard P, Lunec J, Williams A, Good PA, Crewes SJ, Gutteridge JM, Rowley D, Halliwell B, Cornish A, Hider RC: Cerebral and ocular toxicity induced by desferrioxamine. $Q J$ Med 1985, 56(219):345-355.
12. De Virgiliis S, Congia M, Turco MP, Frau F, Dessi C, Argiolu F, Sorcinelli R, Sitzia A, Cao A: Depletion of trace elements and acute ocular toxicity induced by desferrioxamine in patients with thalassaemia. Arch Dis Child 1988, 63(3):250-255.

13. Davies SC, Marcus RE, Hungerford JL, Miller MH, Arden GB, Huehns ER: Ocular toxicity of high-dose intravenous desferrioxamine. Lancet 1983, 2(8343):181-184.

doi:10.1186/1471-2415-14-88

Cite this article as: Wu et al:: Deferoxamine retinopathy: spectral domainoptical coherence tomography findings. BMC Ophthalmology 2014 14:88.

\section{Submit your next manuscript to BioMed Central and take full advantage of:}

- Convenient online submission

- Thorough peer review

- No space constraints or color figure charges

- Immediate publication on acceptance

- Inclusion in PubMed, CAS, Scopus and Google Scholar

- Research which is freely available for redistribution 